\title{
CONTROL EMOCIONAL, FELICIDAD SUBJETIVA Y SATISFACCIÓN VITAL RELACIONADOS AL AFRONTAMIENTO Y ADAPTACIÓN EN PERSONAS CON CÁNCER AVANZADO
}

\section{EMOTIONAL CONTROL, SUBJECTIVE HAPPINESS AND PERSONAL SATISFACTION RELATED TO COPING AND ADAPTATION IN PEOPLE WITH ADVANCED CANCER}

\author{
Alicia Hermosilla Ávila * \\ Olivia SanhueZa Alvarado **
}

\begin{abstract}
RESUMEN
La teoría del Proceso de Afrontamiento y Adaptación de Roy identifica las estrategias que utiliza la persona para responder a las influencias y cambios ambientales que percibe, de manera de crear una integración humana y ambiental. El objetivo de esta investigación fue conocer la relación entre las variables individuales -control emocional, satisfacción vital y felicidad subjetiva- con el proceso de afrontamiento y adaptación al cáncer, en usuarios con diagnóstico de cáncer avanzado del Policlínico del Dolor y Cuidados Paliativos de Chillán. Material y método: estudio descriptivo, transversal y correlacional en una muestra de 49 sujetos inscritos a julio de 2013 en ese centro. Resultados: el 61,2\% de la muestra mostró alta capacidad de afrontamiento y adaptación. Respecto a la dimensión espiritual, el 75,5\% de los participantes presentó alto control emocional, específicamente para la tristeza, siendo mediana para la represión, el enojo y la preocupación. Un 96\% evidenció mayor felicidad subjetiva y alta satisfacción con la vida, existiendo significancia estadística entre la felicidad subjetiva y entre el conocimiento de la patología con el grado de capacidad general de afrontamiento y adaptación. Por su parte la tristeza, como emoción controlada, reveló asociación significativa con el factor 1 recursivo y centrado del proceso de afrontamiento. Asimismo, presentó una relación significativa la variable sexo y el factor 3 de procesos de alerta. Conclusión: algunas de las características de las variables individuales mostraron relación con el grado de afrontamiento y adaptación al cáncer terminal, tales como la felicidad subjetiva y el control emocional de la tristeza.
\end{abstract}

Palabras clave: Adaptación, afrontamiento, cáncer, satisfacción personal, felicidad.

\begin{abstract}
Coping Process theory and Roy Adaptation identifies strategies used by the person to respond to environmental influences and perceived changes so creating a human and environmental integration. The objective of this study was to identify the relationship between the individual variables -emotional control, vital satisfaction and subjective happiness- with the coping and adaptation of cancer in users with a diagnosed advanced cancer, which results contribute with the quality and integrated humanized care in Nursing. Method: A descriptive, transverse and correlate study was developed in 49 samples, enrolled until July 2013. Results: It showed that a

*Enfermera. Docente Depto. de Enfermería. Universidad del Bío-Bío, Chillán, Chile. Email: ahermosilla@ubiobio.cl.

** Enfermera. Docente Facultad de Enfermería. Universidad de Concepción, Concepción, Chile. Email:osanhue@udec.cl.
\end{abstract}


$61,2 \%$ of the samples presented a great capacity of coping and adaptation. According to the spiritual dimension, a high percent of participants presented a high emotional control, specifically in sadness, with a medium control in the repression, anger and concern. A great percent demonstrated a higher subjective happiness and high satisfaction with their lives, existing a statistics significance between subjective happiness and between knowledge of the pathology with the degree of general capacity with the coping and adaptation. On the other hand, the sadness as a controlled emotion, showed a significance association with the factor 1 recursive and centred. As well, it presented a significance relation the sex and the factor 3 from the warning processes. As a conclusion, most participants in this study reflected a high and very high capacity to face the difficulties and problems, in this case, the advanced cancer. In general, the strategies used in the passive and active management adopted a favorable and positive attitude at the moment of taking decisions and solving problems, even in the changes of lifestyle. This shows the relevance in the incorporation of the spiritual dimension aspects of the users in order to get on provision of health quality with proper tools adapted to the social reality. Conclusion: some of the characteristics of individual variables demonstrated relationship with the degree of coping and adaptation of cancer as subjective happiness and emotional control of sadness.

Key words: Adaptation, psychological, coping behavior, cancer, personal satisfaction, happiness.

Fecha recepción: 31/03/14 Fecha aceptación: 06/04/15

\section{INTRODUCCIÓN}

La Organización Mundial de la Salud define al cáncer como un problema global progresivo, con una prevalencia mundial estimada en cerca de 15 millones de casos nuevos, llegando a tener 22,4 millones de personas viviendo con cáncer en cualquier etapa de la enfermedad, desafiando la atención integral del usuario (1). La importancia del cáncer radica en su incidencia o número de nuevos casos que aparecen cada año, además de las cifras de mortalidad por esta causa y el impacto social que ello trae consigo, más aún cuando el cáncer al ser detectado tempranamente es una enfermedad y una patología crónica curable, siendo evidente que ciertos cánceres han aumentado su incidencia pero disminuido su mortalidad (2).

El proceso de afrontamiento al cáncer requiere un esfuerzo integral de la persona, que lo hace vulnerable y dependiente, y genera la necesidad de apoyo emocional para fortalecer la capacidad de resistir los síntomas, signos y cambios en la vida asociados al diagnóstico de cáncer, con una fuerte implicancia en la vida, la muerte, los lazos familiares y las emociones (3), asociaciones que requieren el reconocimiento del enfermero/a de los recursos que dispone la persona para afrontar esta situación compleja de salud y algunas variables de importancia para lograr el bienestar, a pesar del diagnóstico. Estas variables son el control emocional, entendido como la supresión de ciertos sentimientos, que parecen importantes en el conocimiento de sí mismos y en la aceptación de una condición compleja de salud (4); la felicidad subjetiva definida como la evaluación o actitud que cada persona hace sobre su satisfacción en la vida personal (5) y la satisfacción vital, expresada como bienestar psicológico que informa de la respuesta cognitiva sobre su propia vida o existencia, en comparación con sus aspiraciones y logros (6).

El proceso de afrontamiento y adaptación de la persona, teorizado por Callista Roy, requiere construir nuevos conocimientos a través de los recuerdos y experiencias nuevas, que en palabras de Roy es lo que le permite a la persona desarrollar nuevas capacidades o 
destrezas para poder dar solución a problemas o realidades nuevas. También la teoría evoluciona en su concepto de cuidado pasando de una visión de reacción a una visión de reciprocidad, donde la persona ya no es solo un ser biopsicosocial que reacciona ante los estímulos en forma lineal, sino que se les reconoce como seres holísticos, responsables de los procesos de interacción y creatividad, en donde ellos y el mundo tienen patrones y relaciones integrales, y la adaptación ya no está en función de los estímulos, sino como un proceso y resultado, por medio del cual las personas con sentimientos y pensamientos, en forma individual y grupal, utilizan la conciencia consciente y eligen crear una integración humana y ambiental, estando la meta de enfermería enfocada en el apoyo y promoción de la adaptación en la persona, para llegar a su bienestar, teniendo en cuenta los mecanismos y decisiones tomadas para afrontarla (7).

De esta manera, Roy establece que el ambiente está conformado por estímulos, definidos como "todo aquello que provoca una respuesta y que es el punto de interacción del sistema humano con el ambiente", donde la relación estímulo-respuesta permite al profesional de enfermería determinar la prioridad e individualidad de la planificación del cuidado, y de esta manera pueden ajustarse a los cambios que se presentan en el nivel de adaptación, atendiendo a factores comunes que pueden afectarla como son los aspectos culturales, del desarrollo de la persona, los conocimientos y las características del ambiente social y económico en que se desenvuelve, agregando además que la persona posee mecanismos de enfrentamiento para poder hacer frente a los distintos estímulos, a través de procesos innatos y adquiridos de afrontamiento (7).

Si la adaptación incluye diversos procesos, incluidos la habituación emocional y de la atención, modificar el contenido o la estructura de las estrategias de afrontamiento cognitivo ejercerá diferentes efectos en los dis- tintos componentes del bienestar, lo que es determinante en la cotidianidad de un usuario con diagnóstico de cáncer avanzado (8).

Si esa habituación emocional lleva a la inhibición de las emociones, podría además originar otros problemas psicológicos y de salud, por ello James, citado por Morales, hace referencia que no expresar las emociones puede llevar a la persona a un terreno patológico, progresando a la apatía y la enfermedad, como un agente estresor acumulativo, que provocaría trastornos relacionados con el estrés (9).

Entre esas emociones, Carvajal menciona a Leibniz, quien identifica a la felicidad como la satisfacción de todas las necesidades de los seres humanos (10), siendo la satisfacción con la vida una valoración del individuo de su propia vida en términos positivos (11). Ésta conforma el componente cognitivo del bienestar subjetivo, representando la discrepancia percibida entre sus aspiraciones y sus logros, cuyo amplio rango evaluativo va desde la sensación de realización personal hasta la experiencia vital de fracaso o frustración (12).

Se debe considerar a la espiritualidad como una dimensión diferente para cada individuo respecto a su vida, la cual se enraíza en las variables personales y se manifiesta en cómo este afronta y se adapta a las situaciones difíciles. Se puede distinguir algunos patrones comunes en este proceso, más aún cuando la espiritualidad vincula el significado de la vida y su relación con el entorno. Se puede connotar a través de las evaluaciones que la persona hace respecto a su vida personal con la satisfacción o felicidad que vivencia y también arraigado en sus creencias y atributos personales con el manejo y control emocional.

Un estudio en mujeres con cáncer de mama concluyó que las mujeres se adaptaban y afrontaban su problema de salud, mostrando un nivel de adaptación integrado de 59\%, satisfaciendo las necesidades, determinando la significatividad de las relaciones entre los 
procesos de afrontamiento y adaptación con el tipo de diagnóstico y la escolaridad (13). Así también, un estudio descriptivo sobre las estrategias de afrontamiento usadas por las mujeres con diagnóstico y cirugía de cáncer de mama logra, entre sus resultados, agrupar las formas más comunes o predominantes de afrontamiento empleados, en las siguientes categorías: paso a paso, empujando a distancia, lo de siempre, disfrutando de la vida, que trata lo emocional, la preparación para la atención y peores positivo (14).

Respecto al control o represión emocional como contribuyente, en gran medida al pronóstico del cáncer, un estudio español determinó que el control emocional influye de forma significativa y positiva sobre el uso de estrategias activas de afrontamiento al dolor en pacientes oncológicos (15).

En una investigación experimental para la evaluación de la expresión de emociones y bienestar en un grupo de mujeres con cáncer de mama, comprobaron que el autoconocimiento y una buena percepción emocional ayudan a interpretar los sentimientos y emociones, colaborando a una mejor adaptación y al bienestar personal, permitiéndoles sentirse, a pesar del cáncer, más satisfechas consigo mismas y más felices (16).

En cuanto al control de las emociones en pacientes con cáncer, explican en un 39\% el control de la preocupación, 29,1\% el control de la tristeza y en un 26,6\% el control de enfado, encontrando que los que padecían cáncer realizaban más control sobre sus emociones, intentando eliminarlas o suprimirlas más que los sujetos sanos (17).

Considerando que existe aún insuficiente conocimiento de la relación entre los procesos de afrontamiento y adaptación en personas con cáncer avanzado, con algunas variables personales como el control emocional, la felicidad subjetiva y la satisfacción vital, se llevó a cabo este estudio, cuyos resultados podrían permitir entregar un cuidado de enfermería integral y de calidad a las personas adscritas a los policlínicos del dolor y cuida- dos paliativos, basado en una planificación de cuidados individualizados e integradores con objetivos que incorporen la dimensión psicológica y espiritual del paciente, como parte de la comprensión y empatía de la relación transpersonal paciente-enfermero.

Objetivo: Conocer la relación entre las variables personales -control emocional, la felicidad subjetiva y la satisfacción vital-con el proceso de afrontamiento y adaptación al cáncer, en usuarios inscritos en el policlínico del dolor y cuidados paliativos del Hospital Clínico Herminda Martin de Chillán durante el año 2013.

\section{MATERIAL Y MÉTODO}

Este estudio descriptivo, transversal y correlacional fue realizado en usuarios inscritos en el policlínico del dolor y cuidados paliativos con diagnóstico de cáncer avanzado, del Hospital Clínico Herminda Martin de Chillán.

Muestra: Conformada por muestreo no probabilístico casual o incidental, con un total de 49 usuarios, todos inscritos en el policlínico del dolor y cuidados paliativos, que corresponde al 67,12\% del universo. La muestra fue obtenida considerando los criterios de inclusión y exclusión, descartándose al $32,88 \%$ por estar en deterioradas condiciones de salud física y mental, por no acceder la familia a comprometer al usuario en su participación u obtener 3 o más puntos en el Test Minimental de Pfeiffer, lo que se interpreta como sospecha de deterioro cognitivo.

Aspectos e implicancias éticas: Primeramente se solicitó autorización al Comité de Ética de la Facultad de Medicina de la Universidad de Concepción para aprobar la investigación. Posteriormente se solicitó al Comité Ético Científico del Hospital de San Carlos y Hos- 
pital Clínico Herminda Martin, para el acceso a información de los usuarios inscritos y para la recolección posterior de datos, tanto en la realización de la prueba piloto como en el desarrollo del estudio. Posteriormente a cada usuario se le informó personalmente de los objetivos de la investigación, la contribución al estudio, su posibilidad de rechazo a participar y la no obtención de efectos perjudiciales al ser parte de la investigación, solicitando para su participación el consentimiento por escrito, garantizando confidencialidad, privacidad y anonimato de sus datos. Se consideraron los principios éticos de la medicina paliativa, los principios éticos propuestos por Emanuel et al. (18) y las pautas éticas internacionales para la investigación biomédica en seres humanos.

Control de calidad de los instrumentos recolectores de datos: Se realizó una prueba piloto para evaluar comprensión de los instrumentos involucrados en el estudio. Se aplicó en un grupo más reducido de usuarios inscritos formalmente en la unidad de alivio del dolor y cuidados paliativos del Hospital San Carlos en la ciudad de San Carlos, específicamente de 10 inscritos, concluyendo con pequeñas modificaciones del lenguaje para facilitar su comprensión, estimando un tiempo promedio de 20 min de aplicación.

Recolección y análisis de datos: Los datos personales fueron obtenidos de los registros de los usuarios inscritos en el policlínico del dolor y cuidados paliativos del Hospital Clínico Herminda Martin de Chillán, para realizar una entrevista en el domicilio del usuario, donde se aplicó el Cuestionario de antecedentes sociodemográficos y las escalas del estudio.

El instrumento recolector de datos estuvo conformado por un cuestionario de antecedentes sociodemográficos y de salud:

- Escala de Medición del Proceso de Afron- tamiento y Adaptación, que mide la capacidad de afrontamiento y adaptación, desarrollado por Callista Roy en el año 2004 (19), validada su versión en español por Gutiérrez et al. (20). Sarmiento et al. (21) al realizar la validación constructo de la misma Escala, reportaron buena validez de contenido y confiabilidad de 0,70 . Respecto a la fiabilidad de la Escala del Proceso de Afrontamiento y Adaptación en el presente estudio se obtuvo un alpha de Cronbach aceptable de 0,698 para la escala modificada lingüísticamente, con alpha de Cronbach para el factor 1 de 0,65, el factor 2 de 0,75 , el factor 3 de 0,592 , el factor 4 de 0,637 y el factor 5 de 0,637 .

- Escala Courtauld de control emocional elaborada por Watson y Greer, en su versión española validada por Anarte et al. (17) (alpha de Cronbach 0,66).

- Escala de Felicidad Subjetiva desarrollada por Lyubomirsky y Lepper (alpha de Cronbach 0,79) (22).

- Escala de satisfacción con la vida (alpha de Cronbach 0,87), desarrollado por Diener et al., contextualizadas a Latinoamérica por Moyano, en Chile (23).

Los resultados obtenidos en la investigación fueron procesados en el programa estadístico SPSS versión 21, por medio de un análisis descriptivo y correlacional, utilizando tablas de frecuencias para la caracterización de la muestra y chi cuadrado para medir la relación entre las variables, con un nivel de significación $\mathrm{p}<0,05$.

\section{RESULTADOS}

La distribución de los participantes, según los componentes sociodemográficos considerados en el estudio, fue la siguiente: en su mayoría adultos mayores de 65 años, predominando en un $55,1 \%$ el sexo femenino; en un $67,3 \%$ la religión católica; en un $69,4 \%$ 
el estado civil casada/o; el 49\% de los usuarios cursó hasta la enseñanza media; el 42,9\% presentaba dolor moderado y el 22,4\% dolor intenso al momento de recolectar los datos, donde además el 20,4\% no estaba en conocimiento de padecer cáncer.

Respecto del grado de capacidad de afrontamiento y adaptación se encontró que el $61,2 \%$ poseía una alta capacidad, un 32,7\% una muy alta capacidad y un $6,1 \%$ una mediana capacidad, sin evidencia de usuarios con baja capacidad de afrontamiento y adaptación.

En este sentido, respecto a los factores referidos a los comportamientos, recursos, reacciones o estrategias empleadas en estos procesos de afrontamiento y adaptación, el $61,2 \%$ utiliza comportamientos personales y creativos en busca de resultados con una alta capacidad, teniendo esta misma capacidad para afrontar la situación con reacciones físicas en un $51 \%$ y con procesamiento de la información o vivencia personal en un 57,1\%. Asimismo, el 53,1\% tiene una muy alta capacidad de llevar a cabo estrategias metódicas personales y físicas para adaptarse a la situación, y en un 55,1\% utiliza también estrategias propias, que apelan a la memoria y la imaginación, con una muy alta capacidad.

En el control emocional referido por los usuarios respecto a emociones específicas de tristeza, preocupación y enojo, se encontró en el $75,5 \%$ de la muestra un mayor o fuerte control emocional, y un mediano control en el $24,5 \%$ restante, con un control específico de la tristeza en un 61,2\% y del enojo en un $42,9 \%$, destacándose que el 77,6\% también presenta represión de la preocupación.

En la felicidad subjetiva manifestada por los participantes, el 95,9\% refiere mayor felicidad en la actual circunstancia, mientras que el $4,1 \%$ siente menor felicidad, tanto personal como en su percepción comparativa de felicidad de los demás.

En relación a la satisfacción con la vida respecto a su situación actual y sus expectativas en ésta, los participantes señalan alta satisfacción vital en un 98\%, contrastándose con apenas un 2\% con baja satisfacción vital.

En la capacidad de afrontamiento y adaptación de los participantes, según algunas variables sociodemográficas, destaca que quienes poseen una alta capacidad de afrontamiento y adaptación son en un $34,7 \%$ adultos mayores de 65 años; siendo el 36,7\% de ellos de sexo femenino, un 42,9\% profesa la religión católica y el 40,8\% refiere estar casado.

Con respecto a los participantes que conocían su diagnóstico de cáncer, un 46,9\% de ellos presentaba una alta capacidad de afrontamiento y adaptación. Existiendo una relación significativa entre la capacidad de afrontamiento y adaptación con el conocimiento de la patología de cáncer (Chi-cuadrado de Pearson $=15,961 ; p=0,000)$.

Referente a la relación entre el control emocional de los participantes con la capacidad de afrontamiento y adaptación, el 40,8\% presenta alto control o represión emocional en su generalidad, y con alta capacidad de afrontamiento y el $30,6 \%$ lo hace con muy alta capacidad de afrontamiento y adaptación. Destaca que el 34,7\% de quienes tienen alta capacidad de afrontamiento, presentan mediano control de las emociones de enojo y alto control de la tristeza. No se encontró relación significativa entre estas variables.

Respecto a la capacidad de afrontamiento y adaptación y la felicidad subjetiva referida por los participantes, el 59,2\% presentó mayor felicidad con alta capacidad de afrontamiento y adaptación y el 32,7\% presentó mayor felicidad y muy alta capacidad, existiendo entre ellas una relación significativa (Chi-cuadrado de Pearson $=7,280 ; \mathrm{p}=0,026$ ).

Con respecto a la satisfacción con la vida manifestada por los participantes y la capacidad de afrontamiento y adaptación, se encontró que el 59,2\% presentaba alta satisfacción vital, con alta capacidad y el $32.7 \%$ con muy alta capacidad de afrontamiento y adaptación. Sin evidenciar relación significativa entre ambas variables. 
Respecto al factor 1 , que refleja los comportamientos personales y la creatividad en busca de resultados, se observó una relación altamente significativa entre la capacidad de afrontamiento y adaptación y el control emocional (Chi-cuadrado de Pearson $=13,637$; $\mathrm{p}=0,003$ ), específicamente con la tristeza, mostrando que el $32,7 \%$ de los participantes poseen alta capacidad de afrontamiento y adaptación y alto control de la tristeza, asimismo cabe destacar que el 59,2\% que mostraban alta capacidad de afrontamiento y adaptación, presentaban mayor felicidad subjetiva y alta satisfacción con la vida.

En cuanto a los comportamientos enfocados a reacciones físicas o factor 2 , de quienes presentaron alta capacidad de afrontamiento y adaptación, el 36,7\% mostró alto control emocional, el 49\% mayor felicidad subjetiva y el 51\% alta satisfacción con la vida. Respecto la represión de las emociones específicas, el $32,7 \%$ tuvo alta capacidad de afrontamiento y adaptación con mediano control del enojo y el 38,8\% con mediano control de la preocupación. No mostrando relación significativa en ninguna de las variables planteadas.

Los comportamientos vinculados al procesamiento de la información o factor 3 en conjunto con el grado de capacidad de llevar a cabo estrategias de afrontamiento y adaptación muestran, en los usuarios con alta capacidad, un $42,9 \%$ con alto control emocional, un 53,1\% mayor felicidad subjetiva y un $55,1 \%$ alta satisfacción vital. También con alta capacidad, el 30,6\% evidenció alta represión de la tristeza, no encontrándose relación significativa en ninguna de las variables planteadas.

Respecto al manejo metódico de las situaciones o factor 4, los participantes presentaron una muy alta capacidad de afrontamiento y adaptación en asociación con un alto control emocional en un 40,8\%; mayor felicidad subjetiva en un $51 \%$ y alta satisfacción vital en un 53,1\%. Cabe destacar que el $32,7 \%$ de los participantes con muy alta capacidad de afrontamiento y adaptación, tam- bién presentaron mediano control del enojo y el $36,7 \%$ mediano control de la preocupación, sin relación significativa en ninguna de las variables afiliadas.

En relación a los participantes que recurren a sí mismos y a otros, usando la memoria y la imaginación o factor 5, un 44,9\% presenta una muy alta capacidad de afrontamiento y adaptación, mostrando alto control emocional, específicamente en un $40,8 \%$ en la represión de la tristeza. Asimismo el 55,1\% de ellos igualmente refieren mayor felicidad y alta satisfacción. No existiendo relación significativa entre ninguna de las variables.

Respecto a los factores sociodemográficos estudiados y su relación con cada factor de la Escala de Medición del Proceso de Afrontamiento y Adaptación, se destaca la relación significativa entre el conocimiento de la patología con: el factor 1 recursivo y centrado (Chi-cuadrado de Pearson= $13,014 ; \mathrm{p}=0,005)$, factor 2 de reacciones físicas y enfocadas (Chi-cuadrado de Pearson= 19,845; $\mathrm{p}=0,007)$, factor 4 de procesamiento sistemático (Chi-cuadrado de Pearson= 9,706; $\mathrm{p}=0,008$ ) y el factor 5 de conocer y relacionar (Chi-cuadrado de Pearson=11,783; $\mathrm{p}=0,003)$,

En cuanto al factor 3 de procesos de alerta, hubo relación altamente significativa entre el grado de capacidad de afrontamiento y adaptación al cáncer con el conocimiento de su patología de cáncer (Chi-cuadrado de Pearson $=13,637 ; p=0,003$ ) y el sexo (Chi-cuadrado de Pearson $=7,857 ; p=0,049)$.

\section{DISCUSIÓN Y CONCLUSIÓN}

El proceso de afrontamiento y adaptación de las personas sugiere una diversa utilización de comportamientos y estrategias destinadas a la resolución de dificultados o simplemente adecuarse a una situación. Al parecer las personas, a pesar de sus distintas condiciones de vida, situaciones de salud, condición social, 
etc., utilizan patrones de afrontamiento y adaptación similares, caracterizados por una mirada positiva respecto a sus expectativas y actualidad.

Lazcano et al., en México (24), determinaron la capacidad de afrontamiento y adaptación en pacientes crónicos con diagnóstico de diabetes mellitus, encontrando similitudes en la población en promedio de 52 años, mayoritariamente femenino con escolaridad hasta enseñanza media, de aproximadamente 6 años cursados. Respecto al grado de capacidad de afrontamiento y adaptación, a diferencia del presente estudio, encontró una media de 64 puntos equivalentes a baja adaptación, coincidiendo en que los participantes utilizan como comportamiento más común el factor 1, concerniente a la recopilación o investigación de nuevas opciones e información complementaria a sus tratamientos.

Respecto a vivenciar una condición de salud compleja, Flórez et al., en Colombia (25), estudiaron a los pacientes egresados de las unidades de cuidados intensivos determinando que el mayor porcentaje de los pacientes presentaba alta capacidad de afrontamiento y adaptación, al igual que en este trabajo. Igualmente ambos encontraron un mayor porcentaje de participantes vinculados a los comportamientos de alta capacidad para el factor 1, considerando también en la mediana capacidad un mayor porcentaje en el factor 2. Respecto al factor 3 de los egresados de unidades de cuidados intensivos, manifestaron alta capacidad, igual que este estudio. Ambos estudios no presentaron participantes en la categoría de baja capacidad de afrontamiento y adaptación para el factor 5 , cabe considerar que los pacientes del estudio ya estaban inmersos en una situación de cronicidad con apoyo de un equipo multidisciplinario, que difiere de la situación de salud de los usuarios egresados de las unidades críticas de hospitalización.

En el estudio de pacientes oncológicos, Leyva et al., en México (13), determinaron el nivel de afrontamiento y adaptación de 22 mujeres con cáncer de mama, de igual forma este estudio abarca más a mujeres adultas y casadas. Los investigadores tuvieron resultados afines a la presente investigación con respuestas positivas en el $96 \%$ de las usuarias en el factor 1; siendo este factor usado mayoritariamente con una alta capacidad de afrontamiento y adaptación y asimismo en todos los factores debido a que en la valoración general del proceso de afrontamiento y adaptación, conforme con Leyva et al., que refiere que sus participantes muestran altos porcentajes de resolución de problemas analizando detalles, con respuestas positivas, pensando en los problemas sistemáticamente, aclarando todas las dudas para plantear diferentes situaciones; concluyéndose en ambos estudios que la mayoría de los participantes utiliza comportamiento y estrategias combinadas para afrontar y adaptarse a las situaciones conflictivas, difiriendo respecto a la asociación significativa, pues en México se estableció relación con la escolaridad para el factor 1 y 3 , mientras que este estudio evidenció correspondencia en el conocimiento del diagnóstico con todos los factores en concordancia con Moreno-Fergusson y Alvarado García (26), que por medio de una revisión bibliográfica concluyen que el conocimiento de la enfermedad es un estímulo positivo en el proceso.

Otros estudios en pacientes con patologías crónicas, como la insuficiencia renal, fueron efectuados por Del Mar y Pérez, en Colombia (27), en pacientes mayoritariamente masculinos a diferencia de este estudio, también con pacientes adultos de 40 a 70 años, determinando una relación débil entre la perspectiva espiritual y el proceso de afrontamiento y adaptación, donde en el presente estudio sólo se reveló asociación significativa con la felicidad subjetiva referida por los usuarios. En el estudio de Del Mar y Pérez se encontró significación estadística en el proceso de afrontamiento y adaptación con la ocupación para el factor 2, y la escolaridad para el factor 2 y 3 , mientras que en este es- 
tudio sólo hubo asociación significativa con el conocimiento de la patología, la felicidad subjetiva y el sexo, fundamentando la implicancia de esta última en la dimensión espiritual del usuario que indaga precisamente en cómo valora la situación actual y cómo ésta podría evaluarse positivamente gracias a un alto grado de afrontamiento y adaptación al proceso o viceversa.

Los pacientes oncológicos revelan estilos predominantes de afrontamientos que tienen relación con el ir paso a paso con la situación y estar atentos. Además el tema del diagnóstico cobra importancia durante todo el proceso, y promueve el afrontamiento adaptativo, siempre y cuando éste sea tocado con la sensibilidad que amerita, con una visión positiva e incorporando las necesidades individuales para la adaptación final.

En relación a las habilidades de afrontamiento, éstas involucran capacidades no sólo cognitivas sino que también emocionales, siendo importantes la expresión de ayuda familiar y el compartir el dolor como formas de regular la adaptación y el desarrollo psicológico de los pacientes.

En cuanto al control emocional en el pronóstico de cáncer, se evidencia que entre mayor edad existe mayor estilo represivo, donde el control emocional influye significativa y positivamente en las estrategias activas de afrontamiento. En relación a la perspectiva emocional del afrontamiento, se sabe que el autoconocimiento y una buena percepción emocional colaboran con una mejor adaptación y bienestar personal (16), mostrando mayor felicidad subjetiva y alta satisfacción con la vida, considerando que otros estudios señalan que existe más control emocional en pacientes con diagnóstico de cáncer que quienes no lo padecen (28).

Se ha mostrado autorrepresión en la expresión de emociones e incluso afectos, más aún en la exposición de sentimientos como angustia, miedo o malestar, enmascarándolos con otros sentimientos condescendientes con los demás (29), de igual manera en este estudio se mostró control mediano del enojo y un control alto en la tristeza, en contraste con una mayor felicidad subjetiva y manifestaciones de dolor moderado a intenso. Complementando esta información, se ha encontrado un perfil específico de neutralización de reacciones emocionales frente al estadio metastásico de la enfermedad (30), mostrando que al parecer está tan concientizada la enfermedad y condición avanzada, que a pesar de sus molestias asociadas, no hace perder al usuario la perspectiva de proteger a los demás, soportando personalmente el proceso y valorando su vida actual y pasada desde un enfoque positivo y feliz.

Sin embargo, tanto Moyano y Ramos en Chile (31) como Meléndez et al. (32) en España, Diener y Seligman (33) en los Estados Unidos y Edwards y López (34) en México, evidencian que las personas en general se manifiestan más felices, aumentando esta condición si la percepción de salud es alta (35) y más satisfechas con su vida. Vinculándose la mayor satisfacción vital con las actividades cotidianas y la baja percepción de ésta por el estado de salud, dolor y cambios (36); enfatizando todos los estudios en la dimensión social del usuario; concluyendo que las buenas relaciones pueden ser una condición necesaria para la felicidad alta y la satisfacción con la vida.

La manera de enfrentar la vida en cualquier problema o padecimiento crónico en los diversos estudios consultados muestran a usuarios que logran adaptarse adecuadamente y más aún, modelar una mayor felicidad y alta satisfacción con la vida, pero al parecer también controlando algunas emociones que socialmente son poco aceptadas o que pudieran afectar al entorno, oposición que en su conjunto denotan la visión positiva del ser humano a los cambios y desdenes propios de la vida.

La mayoría de los participantes en el presente estudio reflejaron una alta y muy alta capacidad para afrontar y adaptarse a las dificultades o problemas, siendo el cáncer avan- 
zado una de ellas. En cuanto a la relación de las variables individuales y el proceso de afrontamiento y adaptación, se establece una asociación significativa en la muy alta capacidad de desarrollar el proceso con el control de las emociones de tristeza y la percepción de felicidad subjetiva, además de algunos factores sociodemográficos como el sexo y el conocimiento de la patología.

En general, las estrategias utilizadas muestran una actitud favorable y positiva en la toma de decisiones, en los cambios de sus estilos de vida, y en la solución de conflictos, evidenciando lo relevante de la incorporación de los aspectos de la dimensión espiritual del usuario, compatibilizándolas en las prestaciones de salud, con herramientas adecuadas y adaptables a la realidad social, donde la teoría de Callista Roy aporta particularmente a este cuidado de calidad brindado por el profesional de enfermería.

\section{REFERENCIAS}

1. Organización Mundial de la Salud. Prevención de las enfermedades crónicas: una inversión vital [Internet]. Ginebra: OMS; 2005 [citado 10 agosto 2013]. 34 p. Disponible en: http://www.who.int/chp/ chronic_disease_report/overview_sp.pdf

2. Senra A. El cáncer, epidemiología, etiología, diagnóstico y prevención. España: Ediciones Harcourt; 2002. 184 p.

3. Ministerio de Salud Chile. Guía clínica AUGE. Alivio del dolor por cáncer avanzado y cuidados paliativos [Internet]. Santiago: Minsal; 2011 [citado 10 agosto 2013]. 72 p. Disponible en: http://web. minsal.cl/portal/url/item/72213ed52c2723d1e04001011f011398.pdf

4. Lange S. El libro de las emociones: pienso, luego existo. $2^{\text {a }}$ ed. Madrid: Editorial EDAF; 2001. p. 23-25.

5. Galindo J. Ética para adolescentes post modernos. México: Editorial publicacio- nes Cruz O. S.A; 2006. p. 72-73.

6. Leturia F, Yanguas J, Arriola E, Uriarte A. La valoración de las personas mayores: evaluar para conocer, conocer para intervenir. Madrid: Editorial Cáritas Española; $2001.353 \mathrm{p}$.

7. Gutiérrez M, Restrepo L. Aplicación del modelo de adaptación del ciclo vital humano. Colombia: Ediciones Universidad de la Sabana; 2003. p. 16-28.

8. Diener E, Suh E, Lucas R, Smith H. Subjective well-being: three decades of progress. Psychol Bull. 1999; 125 (2): 276302.

9. Morales J, Moya M, Gaviria E, Cuadrado I. Psicología social. $3^{a}$ ed. Madrid: Editorial McGraw Hill; 2007. p. 297-400.

10. Carvajal A. Ética y política en Leibniz parte 1. Revista de Filosofía de la Universidad de Costa Rica. 1999; 37 (91): 69-78.

11. Diener E, Diener M. Cross-cultural correlates of life satisfaction and self-esteem. J Pers Soc Psychol. 1995; 68(4): 653-663.

12. García M. El bienestar subjetivo. Escritos de psicología. 2002; 6: 18-39.

13. Leyva Y, Solano G, Labrador L, Gallegos M, Ochoa M. Nivel de adaptación y afrontamiento en las mujeres con cáncer de mama. Revista Cuidarte. 2011; 2(1): 96-104.

14. Drageset S, Lindstrom T, Underlid K. Coping with breast cancer: between diagnosis and surgery. Journal of advanced nursing. 2009; 66 (1): 149-158.

15. Rodríguez M, Esteve R, López A. Represión emocional y estrategias de afrontamiento en dolor crónico oncológico. Psicothema. 2000; 12 (3): 339- 345.

16. Cerezo M, Cardenal V, Ortiz-Tallo M. Expresión de emociones y bienestar en un grupo de mujeres con cáncer de mama: una intervención psicológica. Rev Latinoam Psicol. 2009; 41 (1): 131-140.

17. Anarte M, Amorós F, Esteve R, López A, Ramírez C. Adaptación al castellano de la escala Cortauld de control emocional en pacientes con cáncer. Psicothema. 2001; 
$13(4): 636-642$.

18. Emanuel EJ, Wendler D, Killen J, Grady C. What makes clinical research in developing countries ethical? The benchmarks of ethical research. J Infect Dis. 2004;189(5):930-7.

19. Roy C, Chayaput P. Coping and Adaptation Processing Scale-English and Thai Versions. Roy Adaptation Association Review. 2004; 6(2):4-6.

20. Gutiérrez C, Crespo O, Dúran M, Moreno M, López C, Veloza M. Validez y confiabilidad de la versión en español del instrumento "Escala de medición del proceso de afrontamiento y adaptación de Callista Roy". Aquichan. 2007; 7 (1): 54- 63.

21. Sarmiento P, Botero J, Carvajal G. Validez de constructo de la Escala de Medición del Proceso de Afrontamiento y Adaptación de Roy, versión modificada en español. Index Enferm. 2013; 22(4): 233-236.

22. Lyubomirsky S, Lepper HS. A measure of subjective happiness: preliminary reliability and construct validation. Soc Indic Res. 1999; 46 (2): 137-55.

23. Diener E, Emmons RA, Larsen RJ, Griffin S. The satisfaction with Life Scale. J Pers Assess. 1985; 49(1): 71-75.

24. Lazcano M, Gómez M, Salazar B. Validación del instrumento: afrontamiento y proceso de adaptación de Roy en pacientes con diabetes mellitus tipo 2. Aquichan. 2008; 8 (1): 116- 125.

25. Flórez I, Herrera E, Carpio E, Veccino M, Zambrano D, Reyes Y, Torres S. Afrontamiento y adaptación en pacientes egresados de unidades de cuidado intensivo. Aquichan. 2011; 11 (1): 23- 39.

26. Moreno-Fergusson M, Alvarado-García A. Aplicación del Modelo de Adaptación de Callista Roy en Latinoamérica: revisión de la literatura. Aquichan. 2009; 9(1): 62-72.

27. Del Mar M, Pérez B. Perspectiva espiritual y proceso de afrontamiento y adaptación en un grupo de pacientes con insuficiencia renal crónica. Revista Cultura del Cuidado de Enfermería. 2011; 1(3): 58-60.

28. Andrés M, Porro-Conforti M. Regulación emocional en personas con y sin diagnóstico de cáncer: Relaciones con ansiedad y depresión. 14º Congreso Virtual de Psiquiatria.com; Feb 2013; Interpsiquis; c2013.

29. Tobo N, Canaval G. Las emociones y el estrés en personas con enfermedad coronaria. Aquichan. 2010; 10 (1): 19-33.

30. Andreu Y, Goldon M, Ibáñez E. Reacciones emocionales a través del estadio en el cáncer de mama. Psicothema. 1991; 3(2): 283-296.

31. Moyano E, Ramos N. Bienestar subjetivo: midiendo satisfacción vital, felicidad y salud en población chilena de la Región del Maule. Revista universum [Internet]. Septiembre 2007 [citado 17 agosto 2011]; 22 (2):177-193. Disponible en: http:// www.scielo.cl/scielo.php?script $=$ sci_arttext\&pid=S0718-23762007000200012\&l$\mathrm{ng}=\mathrm{en} \& \mathrm{nrm}=$ iso\&ignore $=. \mathrm{html}$.

32. Meléndez J, Navarro E, Oliver A, Tomás J. La satisfacción vital en los mayores: factores sociodemográficos. Bol Psicol. 2009; 1(95): 29-42.

33. Diener E, Seligman M. Very happy people. Psychol Sci. 2002; 13 (1): 81-84.

34. Edwards L, López S. Perceived family support, acculturation, and life satisfaction in mexican youth: a mixed-methods exploration. J Couns Psychol. 2006; 53 (3): 279-287.

35. Hernández F, Landero R. Propiedades psicométricas de la escala de felicidad subjetiva (SHS) y su relación con el estrés, la salud percibida y el apoyo social en pacientes con cáncer de mama. Psicooncología. 2014; 11(2-3): 357-367.

36. Cabañero M, Richart M, Cabrero J, Orts M, Reig A, Tosal B. Fiabilidad y Validez de la escala de satisfacción con la vida de Diener en una muestra de mujeres embarazadas y puérperas. Psicothema. 2004; 16 (3): 448-455. 